\title{
Supporting Information: Symmetry-based Crystal Structure Enumeration in Two Dimensions
}

\author{
Evan Pretti, ${ }^{\dagger}$ Vincent K. Shen, ${ }^{\ddagger}$ Jeetain Mittal, ${ }^{\dagger}$ and Nathan A. Mahynski, ${ }^{* \dagger}$ \\ $\dagger$ Department of Chemical and Biomolecular Engineering, Lehigh University, 111 Research \\ Drive, Bethlehem, PA, 18015-4791, USA \\ $\ddagger$ Chemical Sciences Division, National Institute of Standards and Technology, \\ Gaithersburg, MD, 20899-8320, USA \\ E-mail: nathan.mahynski@nist.gov
}




\section{S1 Wallpaper Groups}

A few features of certain wallpaper groups are worth additional consideration. First, note that some variation is permitted in the size and shape of the fundamental domain for certain groups. Naturally, any isotropic scaling may be performed without affecting symmetry, but some domains may be scaled anisotropically as well. For some groups, their side lengths are multiples of a single length scale parameter $L$, while for others, side lengths, $L_{1}$ and $L_{2}$, may be varied independently. For most groups, the angles at the vertices of the domains are fixed, but for $p 1$ and $p 2$, they can be varied as indicated by the parameter $\alpha$. Finally, depending upon the contents of a fundamental domain, degeneracies or overlaps may develop between different wallpaper groups. Perhaps the simplest demonstration of this can be seen by noting that a primitive cell from any of the 17 groups (red parallelograms in Fig. 1 of the main text) is a valid fundamental domain for $p 1$; for example, with luck, one could randomly assign particles inside a $p 1$ fundamental domain such that the pattern has 2-fold rotational symmetry about its midpoint. This would mean the cell actually has $p 2$ symmetry. In general, the constraints imposed by a group require that symmetry no "lower" than itself can be found, but it is possible that we may generate higher symmetry structures. Section S4 examines this in more quantitative detail.

\section{S2 Discretizing Fundamental Domains}

For many groups, the numbers of nodes in each direction, $N_{1}$ and $N_{2}$, may be varied independently with the relative lengths of the two sides, $L_{1}$ and $L_{2}$. A complete list of relative edge lengths and angles used in our generation algorithm is available in the Supporting Information of Ref. 1 so we will not reproduce it, though we briefly review important aspects here. For groups whose sides have equal lengths $L_{1}=L_{2}=L$, we simply have $N_{1}=N_{2}$. For most

groups with triangular domains $L_{1}=L_{2}$ and therefore the grid generated simultaneously enforces that nodes (1) exist along lines drawn parallel to two edges of the domain and (2) 
exist precisely along the diagonal of the domains.

However, in the case of $\mathrm{cmm}$, the FD is triangular and the lengths of its orthogonal edges may vary. To continue to satisfy these two constraints, we must have that $N=\min \left(N_{1}, N_{2}\right)$ along both edges of the parallelogram which generates the lattice; this means that if the edges vary in length, the distance between nodes in each direction will vary as well. Similarly, $p 6 m$ requires unequal orthogonal edge lengths and must also satisfy $N=\min \left(N_{1}, N_{2}\right)$ leading to necessarily unequal spacing along different edges. Thus, the grids for $\mathrm{cmm}$ and $\mathrm{p} 6 \mathrm{~m}$ are independent of $r=L_{2} / L_{1}$; while the former may be freely varied, the latter is constrained to $r=2$. To generate the parallelogram such that nodes exist along the diagonal of these triangular FDs, we must have that $N_{2}=N_{1} \neq\left\lfloor r N_{1}\right\rfloor$ ! 1

\section{S3 Solving the Constrained Symmetry and Stoichiom- etry Problem}

Following the example in Sec. 2.3 of the main text, to ensure that only integers are present, Eq. 2 can be cross-multiplied, and all $c_{j}$ can be multiplied by a suitable factor. Specifically, $Y=\frac{3}{5} Z$ in Eq. 4, which when multiplied by the factor 20, leads to the following expression with integer coefficients after combination with Eq. 5: $20 n_{11}+10 n_{12}+5 n_{13}=12 n_{21}+6 n_{22}+$ $3 n_{23}$.

\section{S4 Redundancy in the CSP}

The similarity between pairs of structures, $S$, was estimated via a cosine similarity based on their radial distribution functions as detailed in Sec. S5. The bin width was set to $\delta r=0.05 \sigma$ and a cutoff distance of $r_{c}=10 \sigma$ was used, where $\sigma=1.0$ is the assumed particle diameter for all species. All structures with $S \geq 0.99$ were considered to be identical. Variation of these parameters did not affect our conclusions. In what follows, we consider all possible 
(a)

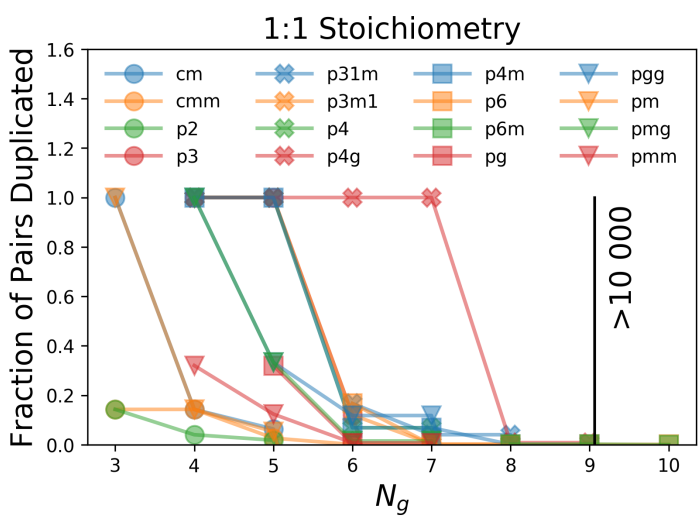

(b)

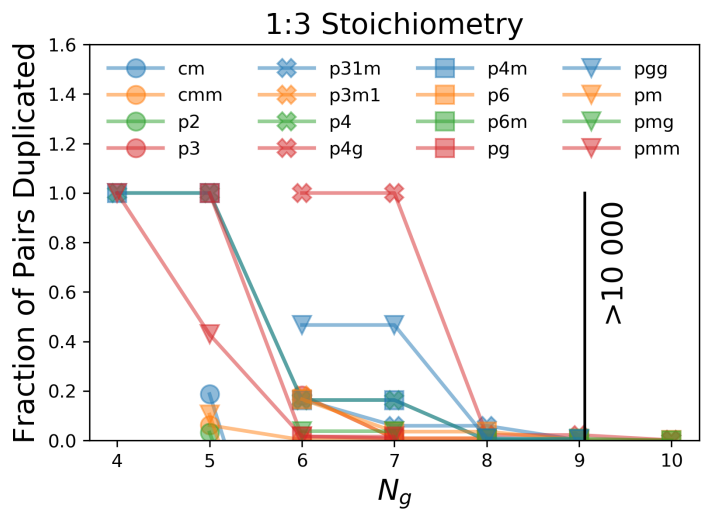

Figure S1: Fraction of unique pairs of configurations considered to be duplicates for each group as $N_{\mathrm{g}}$ increases. For $M$ structures there exist $M(M-1) / 2$ unique pairs. The vertical black line indicates that, across the 16 groups sampled, on average $N_{\mathrm{g}} \approx 9$ is required for the CSP to yield a total of at least $10^{4}$ configurations; for $p 2$ this corresponds to $N_{\mathrm{g}}=6$, for $p 6 m$ this corresponds to $N_{\mathrm{g}}=13$. (a) Results for an equimolar, binary mixture, and (b) a binary mixture with a 1:3 stoichiometric ratio of components.

lattices for each group allowing $r \in(1, \sqrt{2}, \sqrt{3}, 2)$ and $\alpha \in(\pi / 2, \pi / 3, \pi / 4, \pi / 6)$ when these variables are free, otherwise they are fixed to their values required by symmetry. 1

\section{S4.1 Comparison at Fixed Group and Fixed $N_{\mathrm{g}}$}

First, we consider the duplicates that arise from the same lattice, i.e., given a fixed group at a given $N_{\mathrm{g}}$. In the $p 4 g$ group with $\mathrm{A}_{3} \mathrm{~B}_{5}$ stoichiometry example presented in Sec. 2.3 , half of the structures for one chosen solution (ii) to the CSP were duplicates. For a given $N_{\mathrm{g}}$ and group we computed all realizations to all solutions of the CSP and estimated their similarity.

Figure S1 shows the fraction of unique pairs of structures that were found to be duplicates for two representative stoichiometries in a binary system. Although it is not shown explicitly, for sufficiently low $N_{\mathrm{g}}$ there are no identical pairs because the lattice is too small to admit any solutions. However, as $N_{\mathrm{g}}$ increases, the fraction of identical pairs is initially high, often near or equal to unity, and then decays monotonically to zero. In Fig. S1, when the fraction is unity, there exists only a single unique structure among all realizations to all CSP solutions and in general, there are less than 10 total realizations (configurations) in these specific cases. The vertical line in Fig. S1 indicates where, averaged across the groups, we 
must set $N_{\mathrm{g}}$ to achieve at least $10^{4}$ configurations from the CSP. We found that less than $1 \%$ of structure pairs generated for any individual group at this threshold of $N_{\text {config }} \geq 10^{4}$ are redundant for a 1:1 stoichiometry, indicating excellent diversity with this approach.

\section{S4.2 Comparison at Fixed Group with Variable $N_{\mathrm{g}}$}

We must also consider the effect of the number of nodes on the lattice itself. For two primitive cells where one is generated from a lattice with more points than the other, it is not necessarily the case that the solutions found on the larger lattice will contain all solutions found on the smaller one. Scaling the populated lattice to "contact" after a realization to the CSP is generated can help achieve this, but it is not guaranteed. In particular, when the number of lattice nodes along an edge changes from odd to even, sites of special symmetry, like those located at the center of an edge where a $180^{\circ}$ rotation occurs, may appear or disappear. The presence or absence of these special Wyckoff positions on the lattice can create unique structures not found on lattices with a different parity. To understand how well realizations "nest" inside each other as $N_{\mathrm{g}}$ increases we enumerated all lattices for each group as $N_{\mathrm{g}}$ increased until that number exceeded $5 \times 10^{3}$. We then computed the similarity of the lattices found at each $N_{\mathrm{g}}<N_{\mathrm{g}, \max }$ to those at $N_{\mathrm{g}}=N_{\mathrm{g}, \max }$.

Table $\mathrm{S} 1$ lists the number of configurations found for each group given $N_{\mathrm{g}}$ (denominator) and, out of those, the number which are not found $(S<0.99)$ in the ensemble of structures at $N_{\mathrm{g}, \text { max }}$. For example, for $p 2$ with $N_{\mathrm{g}}=3$ there are a total of 8 configurations found, of which all 8 are also found in the 416 found at $N_{\mathrm{g}}=5$. Similarly, all 32 of the configurations which exist when $N_{\mathrm{g}}=4$ are found in those 416 . It is clear that for most of the groups, these configurations nest reasonably well; however, for some, especially those with triangular FDs, there exist instances where some solutions are not found in solutions found at higher $N_{\mathrm{g}}$. This always occurs where the parity (odd-/even-ness) of $N_{1}$ changes, which we attribute to this effect. For example, all 8 configurations for $\mathrm{cmm}$ when $N_{\mathrm{g}}=4$ are found in the 1920 lattices at $N_{\mathrm{g}}=7$, but only 20 out of 128 at $N_{\mathrm{g}}=5$ are contained there. Best practice would 
Table S1: Similarity of structures in an equimolar, binary system for a fixed group as $N_{\mathrm{g}}$ increases. The number of total configurations found up to $N_{\mathrm{g}, \max }$ for each group is given in the denominator. The numerator gives the number of these also found at $N_{\mathrm{g}, \max }$. The table is computed up a given $N_{\mathrm{g}, \max }$, where when $N_{\mathrm{g}} \geq N_{\mathrm{g}, \max }$ the total number of configurations exceeds 5000 for the group. Entries where less than $100 \%$ of the structures are found at $N_{\mathrm{g}, \max }$ are shown in red.

\begin{tabular}{|c|c|c|c|c|c|c|c|c|}
\hline Group $\backslash N_{\mathrm{g}}$ & 3 & 4 & 5 & 6 & 7 & 8 & 9 & 10 \\
\hline $\mathrm{p} 2$ & $8 / 8$ & $32 / 32$ & $416 / 416$ & & & & & \\
\hline $\mathrm{pm}$ & $2 / 2$ & $8 / 8$ & $104 / 104$ & & & & & \\
\hline $\mathrm{pg}$ & $0 / 0$ & $0 / 0$ & $18 / 18$ & $4280 / 4280$ & & & & \\
\hline $\mathrm{pmm}$ & $0 / 0$ & $18 / 18$ & $36 / 36$ & $1574 / 1574$ & $1574 / 1574$ & & & \\
\hline pmg & $0 / 0$ & $2 / 2$ & $4 / 4$ & $326 / 326$ & $326 / 326$ & & & \\
\hline pgg & $0 / 0$ & $2 / 2$ & $4 / 4$ & $38 / 38$ & $38 / 38$ & & & \\
\hline $\mathrm{cm}$ & $2 / 2$ & $8 / 8$ & $104 / 104$ & & & & & \\
\hline $\mathrm{cmm}$ & $8 / 8$ & $8 / 8$ & $20 / 128$ & $1920 / 1920$ & $1920 / 1920$ & & & \\
\hline $\mathrm{p} 4$ & $0 / 0$ & $4 / 4$ & $4 / 4$ & $46 / 46$ & $46 / 46$ & & & \\
\hline $\mathrm{p} 4 \mathrm{~m}$ & $0 / 0$ & $4 / 4$ & $4 / 4$ & $8 / 46$ & $8 / 46$ & $2208 / 2208$ & $2208 / 2208$ & \\
\hline $\mathrm{p} 4 \mathrm{~g}$ & $0 / 0$ & $2 / 2$ & $2 / 2$ & $6 / 6$ & $6 / 6$ & $150 / 150$ & $150 / 150$ & \\
\hline p3 & $0 / 0$ & $6 / 6$ & $6 / 6$ & $60 / 60$ & & & & \\
\hline p3m1 & $0 / 0$ & $6 / 6$ & $6 / 6$ & $12 / 60$ & $2850 / 2850$ & $2850 / 2850$ & & \\
\hline p31m & $0 / 0$ & $0 / 0$ & $0 / 0$ & $0 / 4$ & $84 / 84$ & $84 / 84$ & & \\
\hline p6 & $0 / 0$ & $0 / 0$ & $0 / 0$ & $4 / 4$ & $0 / 18$ & $0 / 18$ & $1892 / 1892$ & $1892 / 1892$ \\
\hline $\mathrm{p} 6 \mathrm{~m}$ & $0 / 0$ & $0 / 0$ & $2 / 2$ & $2 / 2$ & $2 / 2$ & $2 / 32$ & $2 / 32$ & $1566 / 1566$ \\
\hline
\end{tabular}

be to enumerate all solutions for $N_{\mathrm{g}} \leq N_{\mathrm{g}, \max }$ to ensure no structures are missed.

\section{S4.3 Comparison Across Different Groups}

Finally, we consider the possibility that structures created which satisfy the constraints of one group, may also, inadvertently, satisfy those of another. This can happen when nodes of a FD chosen for a configuration form a pattern that is relatively simple. To examine this, we compare the configurations enumerated at $N_{\mathrm{g}, \max }$ for each group. Table S2 shows the fraction of those configurations found in other groups. For each entry at row $i$ and column $j$, the number corresponds to the fraction of configurations in group $i$ found in group $j$. For example, consider the pmm wallpaper group, where we computed 1574 configurations at $N_{\mathrm{g}, \max }=7$ for an equimolar binary mixture ( $c f$. Tab. S1). Only $18 \%$ of these configurations (or 282) are found in the 416 structures calculated for the $p 2$ wallpaper group using $N_{\mathrm{g}, \max }=$ 
5. This table is not symmetric since the number of solutions for group $i$ and $j$ are generally different.

Table S2: Fraction of structures at $N_{\mathrm{g}, \max }$ from Tab. S1 duplicated between groups. Row $i$ and column $j$ corresponds to the fraction of structures for group $i$ at its $N_{\mathrm{g}, \max }(i)$ that are found in group $j$ 's structures found at $N_{\mathrm{g}, \max }(j)$. The vertical and horizontal lines split the table at a pivot point which divides the groups into those with and without hexagonal Bravais lattices.

\begin{tabular}{lrrrrrrrrrrr|rrrrr}
\hline & $\mathrm{p} 2$ & $\mathrm{pm}$ & $\mathrm{pg}$ & $\mathrm{pmm}$ & $\mathrm{pmg}$ & $\mathrm{pgg}$ & $\mathrm{cm}$ & $\mathrm{cmm}$ & $\mathrm{p} 4$ & $\mathrm{p} 4 \mathrm{~m}$ & $\mathrm{p} 4 \mathrm{~g}$ & $\mathrm{p} 3$ & $\mathrm{p} 3 \mathrm{~m} 1$ & $\mathrm{p} 31 \mathrm{~m}$ & $\mathrm{p} 6$ & $\mathrm{p} 6 \mathrm{~m}$ \\
\hline $\mathrm{p} 2$ & 1.00 & 0.25 & 0.38 & 0.38 & 0.25 & 0.12 & 0.12 & 0.08 & 0.04 & 0.04 & 0.04 & 0.00 & 0.00 & 0.00 & 0.00 & 0.00 \\
$\mathrm{pm}$ & 0.98 & 1.00 & 1.00 & 1.00 & 1.00 & 0.31 & 0.31 & 0.15 & 0.13 & 0.13 & 0.13 & 0.00 & 0.00 & 0.00 & 0.00 & 0.00 \\
$\mathrm{pg}$ & 0.06 & 0.06 & 1.00 & 0.20 & 0.06 & 0.03 & 0.03 & 0.04 & 0.02 & 0.05 & 0.03 & 0.00 & 0.00 & 0.00 & 0.00 & 0.00 \\
$\mathrm{pmm}$ & 0.18 & 0.17 & 0.39 & 1.00 & 0.25 & 0.08 & 0.09 & 0.03 & 0.08 & 0.03 & 0.03 & 0.00 & 0.00 & 0.00 & 0.00 & 0.00 \\
$\mathrm{pmg}$ & 0.32 & 0.33 & 0.35 & 0.55 & 1.00 & 0.14 & 0.17 & 0.06 & 0.07 & 0.05 & 0.05 & 0.00 & 0.00 & 0.00 & 0.00 & 0.00 \\
$\mathrm{pgg}$ & 0.63 & 0.58 & 0.68 & 1.00 & 0.89 & 1.00 & 1.00 & 0.37 & 0.21 & 0.21 & 0.21 & 0.00 & 0.00 & 0.00 & 0.00 & 0.00 \\
$\mathrm{~cm}$ & 0.25 & 0.23 & 0.27 & 0.58 & 0.54 & 0.50 & 1.00 & 0.15 & 0.10 & 0.10 & 0.10 & 0.00 & 0.00 & 0.00 & 0.00 & 0.00 \\
$\mathrm{cmm}$ & 0.02 & 0.01 & 0.04 & 0.03 & 0.01 & 0.03 & 0.03 & 1.00 & 0.01 & 0.05 & 0.04 & 0.00 & 0.00 & 0.00 & 0.00 & 0.00 \\
$\mathrm{p} 4$ & 0.17 & 0.17 & 0.48 & 1.00 & 0.26 & 0.17 & 0.17 & 0.17 & 1.00 & 0.17 & 0.17 & 0.00 & 0.00 & 0.00 & 0.00 & 0.00 \\
$\mathrm{p} 4 \mathrm{~m}$ & 0.01 & 0.01 & 0.02 & 0.01 & 0.01 & 0.01 & 0.01 & 0.08 & 0.01 & 1.00 & 0.07 & 0.00 & 0.00 & 0.00 & 0.00 & 0.00 \\
$\mathrm{p} 4 \mathrm{~g}$ & 0.04 & 0.04 & 0.11 & 0.04 & 0.04 & 0.04 & 0.04 & 0.39 & 0.04 & 0.45 & 1.00 & 0.00 & 0.00 & 0.00 & 0.00 & 0.00 \\
\hline $\mathrm{p} 3$ & 0.00 & 0.00 & 0.00 & 0.00 & 0.00 & 0.00 & 0.00 & 0.00 & 0.00 & 0.00 & 0.00 & 1.00 & 0.20 & 0.20 & 0.20 & 0.10 \\
$\mathrm{p} 3 \mathrm{~m} 1$ & 0.00 & 0.00 & 0.00 & 0.00 & 0.00 & 0.00 & 0.00 & 0.00 & 0.00 & 0.00 & 0.00 & 0.00 & 1.00 & 0.02 & 0.03 & 0.02 \\
$\mathrm{p} 31 \mathrm{~m}$ & 0.00 & 0.00 & 0.00 & 0.00 & 0.00 & 0.00 & 0.00 & 0.00 & 0.00 & 0.00 & 0.00 & 0.07 & 0.57 & 1.00 & 0.00 & 0.50 \\
$\mathrm{p} 6$ & 0.00 & 0.00 & 0.00 & 0.00 & 0.00 & 0.00 & 0.00 & 0.00 & 0.00 & 0.00 & 0.00 & 0.00 & 0.00 & 0.00 & 1.00 & 0.00 \\
$\mathrm{p} 6 \mathrm{~m}$ & 0.00 & 0.00 & 0.00 & 0.00 & 0.00 & 0.00 & 0.00 & 0.00 & 0.00 & 0.00 & 0.00 & 0.00 & 0.01 & 0.01 & 0.00 & 1.00 \\
\hline
\end{tabular}

Notably, the table contains a pivot point between $p 4 g$ and $p 3$. It is clear that this corresponds to a split between the first two rows and the third row of Fig. 2 in the main text. Groups in the third row $(p 3-p 6 m)$ correspond to hexagonal Bravais lattices, while the first two rows correspond to non-hexagonal Bravais lattices. ${ }^{2}$ Thus, lattices which are hexagonal tend to be most similar to other hexagonal lattices, and vice-versa, consistent with intuition. While the table reflects all zeros for these "cross-lattice" comparisons (hexagonal vs. non-hexagonal) for an equimolar mixture, overlap at other stoichiometries may be finite, but is generally small. Figure S2 shows a row-wise average (neglecting diagonal entries which are all 1) of Tab. S2. Two other stoichiometries are also considered; note that the groups with the most overlap in an equimolar system do not necessarily correspond to those in systems with a different stoichiometry.

Averaging over all groups with an equal weight suggests a mean, average overlap of 


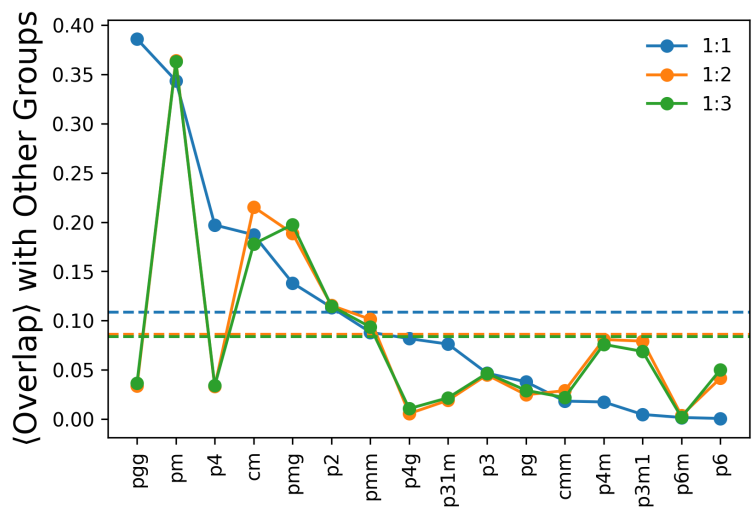

Figure S2: Average fractional overlap for each group with respect to the other 15 considered for binary systems. This amounts to a row-wise average of Tab. S2, neglecting the diagonal, for the equimolar mixture. 1:2 and 1:3 stoichiometries are also considered. Dashed lines correspond to an average of the average overlap for each stoichiometry.

roughly $10 \%$, regardless of stoichiometry. To maintain diversity in practice, we generally sample a fixed number of structures from each group, equally weighting each group's contribution to the overall ensemble of configurations generated. However, one could also sample randomly from all structures generated across all groups at a fixed $N_{\mathrm{g}}$. In Fig. S2, $p g g$ dominates the mean overlap for an equimolar mixture; i.e., there are only 38 structures generated at $N_{\mathrm{g}, \max }=7$ ( $c f$. Tab. S1), yet those structures can often be generated by our algorithm in other groups. However, for a fixed $N_{\mathrm{g}}$ the groups $p 2, p m$, and $\mathrm{cm}$ tend to dominate the overall number of configurations that can be found. Assuming the average overlap is dominated by contributions from these three groups instead suggests a mean, average overlap of $20 \%$ to $30 \%$.

\section{S5 Estimating Structural Similarity}

A method for measuring the similarity between structures is required to identify duplicate structures. We have chosen a method similar to one proposed in Ref. 3 based on comparing the similarities of pairwise radial distribution functions (RDFs). For two structures 
designated $\alpha$ and $\beta$, we define the (cosine) similarity for particle types $i$ and $j$ as:

$$
S_{i j}^{\alpha \beta}=\frac{\sum_{0}^{r_{c}} g_{i j}^{\alpha}(r) g_{i j}^{\beta}(r)}{\sqrt{\sum_{0}^{r_{c}}\left[g_{i j}^{\alpha}(r)\right]^{2}} \sqrt{\sum_{0}^{r_{c}}\left[g_{i j}^{\beta}(r)\right]^{2}}}
$$

where $g_{i j}$ is the pairwise RDF histogram between particles of type $i$ and those of type $j$. $S_{i j}^{\alpha \beta}$ takes on a maximum value of 1 if $g_{i j}^{\alpha}(r)=g_{i j}^{\beta}(r)$ for all $r \in\left[0, r_{c}\right]$, and a minimum value of 0 if there exists no overlap between the non-zero regions of $g_{i j}^{\alpha}(r)$ and $g_{i j}^{\beta}(r)$. We define the similarity between the structures as $S^{\alpha \beta}=\min _{i, j}\left(S_{i j}^{\alpha \beta}\right)$ so that two structures can only be as similar as their least similar pairwise distribution. In this way $0 \leq S^{\alpha \beta} \leq 1$, where $S^{\alpha \beta}=1$ indicates that all pairwise RDFs match exactly.

If pairwise distances are accumulated directly into individual bins, numerical issues will arise if two nearly identical structures happen to have pairwise distances close to a multiple of $\delta r$, as slightly different rounding behavior may cause particles to be split arbitrarily between adjacent bins. To resolve this, smoothing can be applied by interpolating the contribution of a given pairwise distance into the two nearest bins based on its relative proximity to the boundary between them. Once $S^{\alpha \beta}$ has been calculated, two structures can be said to be equivalent if and only if $S^{\alpha \beta}$ exceeds a selected cutoff value. We have found for the colloidal systems tested with our framework that this measure works well for distinguishing structures, although other methods based on, e.g., direct comparison of the periodic structures ${ }^{\sqrt{4}}$ could $^{-}$ be used as well. 


\section{References}

(1) Mahynski, N. A.; Pretti, E.; Shen, V. K.; Mittal, J. Using symmetry to elucidate the importance of stoichiometry in colloidal crystal assembly. Nat. Commun. 2019, 10, 2028.

(2) Schattschneider, D. The plane symmetry groups: their recognition and notation. The American Mathematical Monthly 1978, 85, 439-450.

(3) Oganov, A. R.; Valle, M. How to quantify energy landscapes of solids. J. Chem. Phys. 2009, 130, 104504.

(4) Hundt, R.; Schön, J. C.; Jansen, M. CMPZ - an algorithm for the efficient comparison of periodic structures. J. Appl. Crystallogr. 2006, 39, 6-16. 